\title{
Cardiovascular Health in Anxiety or Mood Problems Study (CHAMPS): study protocol for a randomized controlled trial
}

Phillip J. Tully ${ }^{1,2,8^{*}}$, Deborah A. Turnbull3,8, John D. Horowitz ${ }^{4}$, John F. Beltrame ${ }^{4}$, Terina Selkow ${ }^{4}$, Bernhard T. Baune ${ }^{5}$, Elizabeth Markwick ${ }^{6}$, Shannon Sauer-Zavala ${ }^{7}$, Harald Baumeister ${ }^{1}$, Suzanne Cosh $^{3}$ and Gary A. Wittert ${ }^{8}$

\begin{abstract}
Background: Previous psychological and pharmacological interventions have primarily focused on depression disorders in populations with cardiovascular diseases (CVDs) and the efficacy of anxiety disorder interventions is only more recently being explored. Transdiagnostic interventions address common emotional processes and the full range of anxiety and depression disorders often observed in populations with CVDs. The aim of CHAMPS is to evaluate the feasibility of a unified protocol (UP) for the transdiagnostic treatment of emotional disorders intervention in patients recently hospitalized for CVDs. The current study reports the protocol of a feasibility randomized controlled trial to inform a future trial.

Methods/Design: This is a feasibility randomized, controlled trial with a single-center design. A total of 50 participants will be block-randomized to either a UP intervention or enhanced usual care. Both groups will receive standard CVD care. The UP intervention consists of 1) enhancing motivation, readiness for change, and treatment engagement; (2) psychoeducation about emotions; (3) increasing present focused emotion awareness; (4) increasing cognitive flexibility; (5) identifying and preventing patterns of emotion avoidance and maladaptive emotion-driven behaviors (EDBs, including tobacco smoking, and alcohol use); (6) increasing tolerance of emotion-related physical sensations; (7) interoceptive and situation-based emotion-focused exposure; and (8) relapse prevention strategies. Treatment duration is 12 to 18 weeks. Relevant outcomes include the standard deviation of self-rated anxiety, depression and quality of life symptoms. Other outcomes include intervention acceptability, satisfaction with care, rates of EDBs, patient adherence, physical activity, cardiac and psychiatric readmissions. Parallel to the main trial, a nonrandomized comparator cohort will be recruited comprising 150 persons scoring below the predetermined depression and anxiety severity thresholds.
\end{abstract}

Discussion: CHAMPS is designed to evaluate the UP for the transdiagnostic treatment of emotional disorders targeting emotional disorder processes in a CVD population. The design will provide preliminary evidence of feasibility, attrition, and satisfaction with treatment to design a definitive trial. If the trial is feasible, it opens up the possibility for interventions to target broader emotional processes in the precarious population with CVD and emotional distress.

Trial registration: ACTRN12615000555550, registered on 29/05/2015

Keywords: Depression, major depression, anxiety, anxiety disorders, cardiovascular disease, coronary heart disease, randomized controlled trial, cognitive behavioral therapy, diagnosis, prognosis

\footnotetext{
* Correspondence: phillip.tully@adelaide.edu.au

${ }^{1}$ Department of Rehabilitation Psychology and Psychotherapy, Institute of

Psychology, University of Freiburg, Engelbergerstr. 41, Freiburg 79085,

Germany

${ }^{2}$ INSERM, U897-Epidemiology and Biostatistics, Bordeaux, France

Full list of author information is available at the end of the article
} 


\section{Background}

Depression disorders are severely disabling and common in cardiovascular disease (CVD) populations and portend poorer cardiovascular outcome [1, 2] and high costs [3]. Consequently, the treatment of depression disorders has dominated the contemporary psychological intervention landscape in populations with CVD for the past 25 years. Considering that depression is a putative CVD risk factor that is also modifiable, a common hypothesis is that psychosocial interventions in this population would lead to significant benefits to an otherwise poorer cardiovascular prognosis. However, prior intervention efforts addressing depressive symptoms among CVD patients have not typically produced a sizeable clinical impact upon either depression or major CVD morbidity [4], and these unsatisfying findings raise the question of how existing interventions could be improved.

Several lines of evidence indicate that interventions focusing solely on depressive symptoms in CVD patients may be too narrow in focus. Specifically, an accumulating body of work shows that anxiety disorders confer CVD morbidity risk independent of or in conjunction with depression [5-14]. Indeed, the clinical reality of comorbid depression and anxiety that is evident in CVD populations [15] closely parallels findings in clinical and epidemiological samples $[16,17]$. Nonetheless, the heightened CVD risk is not constrained to simply depression and anxiety disorders given that positive associations between CVD events have been reported in relation to hostility, anger, stress, social isolation, worry, rumination, somatic depressive symptoms, anxiety sensitivity, phobic anxiety and the specific combination of negative affectivity and social inhibition [15, 18-22]. Collectively these findings point to the likelihood that common processes underlying negative emotions generally also portend CVD risk [19], raising the possibility that an intervention that transcends diagnostic boundaries and targets core emotional processes would be a step toward improving mental health interventions among CVD populations.

\section{Limitations of the extant depression intervention findings in CVD}

Several lines of evidence raise the possibility that interventions focusing solely on depression disorders have been ineffective in the population with CVDs. First, prior controlled efforts to address depression in patients with coronary artery disease [4, 23] and heart failure [24] via psychological and pharmacological interventions have reported only small, albeit significant, effects on depression symptoms. Strikingly, the effect sizes for depression symptom reduction are markedly smaller in CVD samples than that observed in other chronic diseases such as Type II diabetes samples [25]. Collective findings have prompted a closer examination of the specific therapeutic components of interventions for depression used in CVD samples that might lead to an improved outcome [26], as well as an exploration of novel methods of mental health service delivery such as collaborative care [27]. Intriguingly, neither avenue of empirical enquiry has consistently yielded moderate or larger effect sizes for depression symptom reduction to date. Likewise, despite initially promising findings from one collaborative care randomized controlled trial (RCT) suggesting a reduction in major adverse cardiac events (MACE) [28], these findings were not sustained in the longer term [23, 29]. Together, these findings point to the strong possibility that mental health interventions exclusively targeting depression in CVDs are incomplete and too narrow in focus.

\section{Anxiety burden and effects on CVD prognosis}

Parallel to what has been reported for depression, an emerging literature has uncovered an association between anxiety with CVD morbidity [8, 15], prompting questions regarding whether depression is a discrete psychiatric risk factor for MACE [11, 15]. Specifically, two recent meta-analyses indicated that post-traumatic stress disorder was associated with a twofold recurrent acute coronary syndrome risk [7] whereas generalized anxiety disorder (GAD) was associated with a $21 \%$ increase in MACE risk [15]. Although the association between panic disorder and CVD events is tenuous [9, 30-34], panic disorder nonetheless signifies high emergency department utilization for chest pain and diagnostic tests to rule out acute coronary syndromes [35], representing a major burden on the healthcare system.

\section{Importance of anxiety intervention in CVDs}

Alongside depression, the American Heart Association [36] and the German Cardiac Society [21] recommend comprehensive assessment of anxiety disorders in CVD patients. In depressed CVD patients, the prevalence of comorbid anxiety disorders is $30 \%$ to $50 \%[15,27]$. In addition, $50 \%$ of cardiac patients presenting for psychotherapy meet at least one anxiety disorder diagnosis [37, 38], and higher anxiety is associated with depression treatment resistance [39]. Despite the prevalence of anxiety disorders in CVDs exceeding community estimates [17] and comparable to depression prevalence in CVD [15], no clinical guidelines exist for treatment when anxiety disorder comorbidity is present in this population [40]. Moreover, with increasing recognition of the necessity to formulate interventions matching the complexity of CVD populations [37, 41] and the requirement for mental health interventions to match transdiagnostic developments in clinical psychology [42-47], the use of depression disorder-only treatments may become increasingly obsolete. 


\section{Previous work supporting the need for a transdiagnostic approach in CVDs}

Our recent work supports the assertion that an expansion of mental health interventions beyond depression to incorporate comorbid anxiety disorders is warranted in populations with CVDs. A screen-and-treat model of care for heart failure patients was undertaken in three South Australian tertiary hospitals where we observed that panic disorder and anxiety due to a medical condition was associated with high emergency department utilization [48]. Furthermore, a reduction in CVD readmissions was observed with CBT for GAD [49]. We hypothesized that an intervention focusing on somatic anxiety symptoms led to a significant reduction in CVD hospital readmissions and to an improved depression response [49].

Despite strong evidence collectively demonstrating how anxiety disorders are common and necessitate treatment in CVDs, a critical absence of interventions with broader reach beyond depression exists, which forms a major oversight in improving upon existing mental health interventions. Therefore, treatment of a broader range of emotional disorders and their common emotional processes likely would be an innovative step toward improving mental health interventions and cardiovascular health among CVD populations. Targeting the most common psychological processes that confer CVD risk with a single set of therapeutic principles would open the possibility for a more parsimonious application of effective mental health interventions to a broader range of CVD patients. Herein, we outline the methodology for a study of this type based on the unified protocol (UP) for transdiagnostic treatment of emotional disorders [42, 44-46].

\section{Study objectives}

The aim of the Cardiovascular Health in Anxiety and Mood Problems Study (CHAMPS) is to prospectively study the UP in patients with a recent CVD hospitalization and comorbid depression and/or anxiety. The outcomes of interest include the standard deviation of self-rated depression, anxiety and quality of life symptoms. Other relevant outcomes include rates of EDBs (tobacco smoking and alcohol use), physical activity levels, patient adherence, cardiac and psychiatric readmissions. A third objective is to explore trajectories of emotional distress in relation to EDBs and psycho-behavioral CVD risk factors. The data obtained will provide an estimate of effect size for a larger more definitive trial.

\section{Methods}

\section{Study design}

This prospective study is a feasibility randomized controlled trial, of parallel design, comparing the effectiveness of the UP versus enhanced usual care. A total of 50 participants will be recruited from the Queen Elizabeth Hospital, a tertiary hospital in the western urban area of Adelaide, South Australia. Parallel to the main trial, a nonrandomized comparator cohort will be recruited comprising at least 150 persons scoring below the predetermined depression and anxiety severity thresholds. Figure 1 depicts the trial design. The trial will be conducted in accordance with the CONSORT statement [50].

\section{Inclusion criteria}

Inclusion criteria are as indicated below:

1. Age $\geq 18$ years.

2. A primary hospital admission for CVD (specified by relevant International Classification of Disease codes for coronary artery disease, myocardial infarction, heart failure, atrial fibrillation, other ventricular or atrial arrhythmia, coronary revascularization intervention, symptomatic coronary heart disease including unstable angina pectoris, or heart valve disease).

3. A MINI International Neuropsychiatric Interview (MINI) diagnosis by randomization naïve assessors of major depression, dysthymia, GAD, panic disorder, agoraphobia, social anxiety/phobia, or post-traumatic stress disorder.

4. Above the severity threshold for depression PHQ scores $(\geq 10)[51]$ or anxiety threshold GAD scores $(\geq 7)[52]$.

5. Fluency in English.

\section{Exclusion criteria}

Exclusion criteria include the following:

- A psychosis or bipolar disorder diagnosis determined by medical history or randomizationnaïve assessors.

- High suicide risk at psychiatric interview.

- Observed cognitive impairment or dementia impeding delivery of psychotherapy or ability to provide informed consent.

- Neurodegenerative condition (for example, Parkinson's disease or multiple sclerosis)

- In receipt of GP, psychologist or psychiatrist counselling elsewhere.

- A diagnosis of drug and alcohol dependence or abuse determined by randomization-naïve assessors.

- Medical condition likely to be fatal within 1 year.

\section{Nonrandomized comparator cohort}

Participants scoring below the depression and anxiety severity threshold on the PHQ-9 and GAD-7, and therefore those without any distress, will be part of a nonrandomized comparator cohort. The rationale for the comparator 


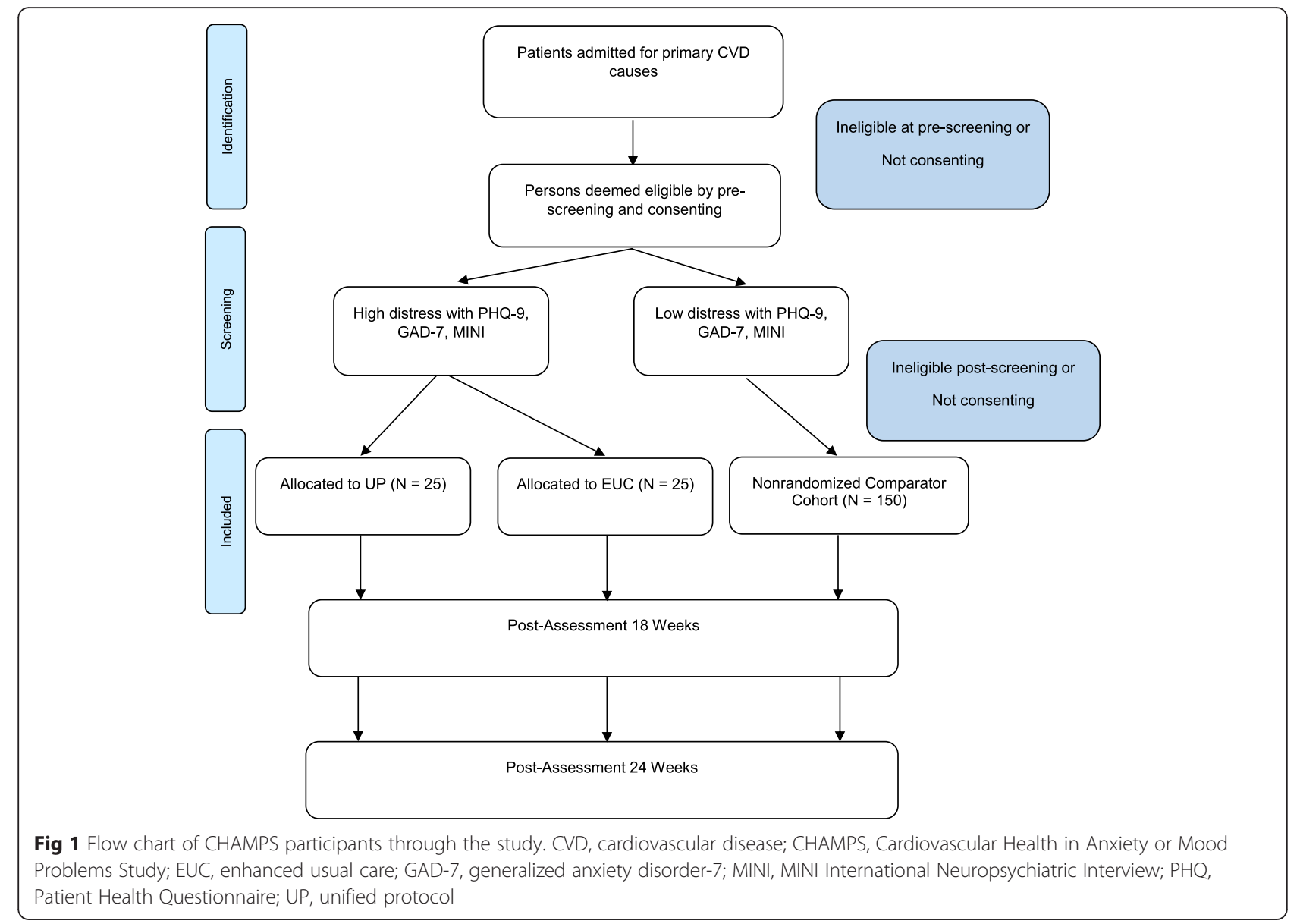

cohort is to answer empirical questions about EDBs, incident emotional disorders and trajectories of emotional distress. Several recent RCTs have adopted a similar approach and recruited a comparator cohort for observational purposes.

\section{Non-randomized comparator cohort eligibility}

1. Age $\geq 18$ years.

2. A primary hospital admission for CVD (specified by relevant International Classification of Disease codes for coronary artery disease, myocardial infarction, heart failure, atrial fibrillation, other ventricular or atrial arrhythmia, coronary revascularization intervention, symptomatic coronary heart disease including unstable angina pectoris, or heart valve disease).

3. Free from any MINI diagnosis by randomization-naïve assessors of major depression, dysthymia, GAD, panic disorder, agoraphobia, social anxiety/phobia, and post-traumatic stress disorder.

4. Free from any MINI diagnosis by randomization-naïve assessors or medical history of psychosis or bipolar disorder.
5. Below the severity threshold for depression PHQ scores $(\leq 9)$ [51] or anxiety threshold GAD scores $(\leq 6)[52]$.

6. Fluency in English.

\section{Proposed sample}

The proposed sample for this study was 50 patients randomized to the UP or EUC arm. Parallel to the RCT, 150 persons will be recruited into the nonrandomized comparator cohort. In the total sample $(N=200)$, incorporation of the nonrandomized comparator cohort $(n=150)$ and RCT participants $(n=50)$ will enable us to explore the rates of EDBs, physical activity, medication adherence, hospital readmission and feasibility of a screen-and-treat model of care.

\section{Planned statistical analysis}

Data management is outlined elsewhere in our protocol. Comparisons between UP, EUC and the nonrandomized comparator cohort will be made at each follow-up time point on patient level of satisfaction with aspects of psychosocial care. We will evaluate whether screening, education, and support in EUC is a suitable alternative to UP. We will also monitor linkages with primary care to 
determine whether our suicide risk management strategy is viable when routine depression screening is implemented. These data will help inform the design and dayto-day running of a larger more definitive RCT. In terms of feasibility, we will also evaluate the recruitment eligibility, acceptance, and attrition rates, the length of time taken to recruit, and compliance with the psychosocial questionnaire battery.

In the total sample, incorporating the nonrandomized comparator cohort, the association between EDBs, physical activity, and medication adherence with readmission will be examined. Incident psychological distress and distress trajectories will be evaluated in multi-level models to provide a snapshot of different distress trajectories on each of the measures used in CHAMPS. The longitudinal changes in QOL will also be analyzed in the same manner as incident psychological distress and distress trajectories described above.

\section{Randomization}

An independent statistician will generate the randomization codes. Patients will be block randomized according to a random number generator in alternating block sizes of three and six. Allocation will be concealed in sequentially numbered, opaque, sealed envelopes. Randomization will be stratified by primary CVD admission (myocardial infarction, heart failure, atrial fibrillation, other ventricular or atrial arrhythmia, coronary revascularization intervention, symptomatic CHD including angina pectoris, and heart valve disease) to obtain approximately equal sample sizes in both groups.

\section{Transdiagnostic unified protocol intervention}

The UP is a transdiagnostic cognitive-behavioral intervention explicitly designed to address the full range of emotional disorders (anxiety, depressive, and related disorders); this work is done by targeting core, underlying emotional processes that lead to the development and maintenance of symptoms across disorders. The UP is designed for weekly and face-to-face delivery over 12 to 18 sessions. The UP consists of eight modules: (1) enhancing motivation for change and treatment engagement, (2) facilitating better understanding of patients' emotional experiences, (3) increasing present focused emotion awareness, (4) increasing cognitive flexibility, (5) identifying and preventing patterns of emotion avoidance and maladaptive EDBs, (6) increasing awareness and tolerance of emotion-related physical sensations, and (7) interoceptive and situation-based emotion-focused exposure, and (8) the final module is devoted to summarizing the relevant techniques attained and to developing relapse prevention strategies. A summary of the UP decision tree and intervention outline is shown in Table 1. A more complete description by Barlow et al. [53] can be found elsewhere regarding the development of the UP and longterm treatment outcomes. An experienced psychologist, who is trained in the UP and trained by the research team on CVDs, will deliver the intervention. In psychotherapy intervention trials, blinding of the study participants, therapists, and study coordinator is not possible. However, all other members of the research team will remain blinded. Intervention fidelity will be maintained by weekly supervision and monitored to determine if the UP requires modification in a larger trial among CVD patients.

\section{Enhanced usual care}

Patients randomized to the EUC group will receive an education package delivered by the study coordinator consisting of the beyondblue ${ }^{\mathrm{Ts}}$ fact sheet regarding anxiety, depression, and coronary heart disease [54]. Participants and their general physician will be informed of the baseline distress results and directed to available clinical services (psychologist or psychiatrist), advising participants to seek assistance for achieving mental wellbeing

Table 1 A Description of the eight modules for the transdiagnostic unified protocol in the intervention group

\begin{tabular}{|c|c|c|}
\hline & & Transdiagnostic unified protocol \\
\hline Module & Session & Content \\
\hline 1. Preliminary module & 1 & $\begin{array}{l}\text { Focusing on enhancing motivation and readiness for change and treatment } \\
\text { engagement }\end{array}$ \\
\hline 2. Psychoeducation & 2 & $\begin{array}{l}\text { Educating patients on the nature of emotions and providing a framework for } \\
\text { understanding their emotional experiences }\end{array}$ \\
\hline 3. Present focused awareness & $3-4$ & Increasing present focused emotion awareness \\
\hline 4. Cognitive flexibility & $5-7$ & Increasing cognitive flexibility \\
\hline 5. Emotion-driven behaviors & $8-11$ & $\begin{array}{l}\text { Identifying and preventing patterns of emotion avoidance and maladaptive } \\
\text { emotion-driven behaviors }\end{array}$ \\
\hline 6. Emotion awareness and tolerance & $12-15$ & Increasing awareness and tolerance of emotion-related physical sensations \\
\hline 7. Exposure & $16-17$ & Interoceptive and situation-based emotion focused exposure \\
\hline 8. Summary & 18 (or earlier if required) & $\begin{array}{l}\text { Summarizing the relevant techniques attained and developing relapse prevention } \\
\text { strategies. }\end{array}$ \\
\hline
\end{tabular}


with the support of their general physician. This conforms to the National Heart Foundation of Australia's $\mathrm{s}^{\mathrm{m}}$ guidelines $[51,55]$. There are no restrictions on usual care.

\section{Standard of care}

All participants will receive standard medical care for CVDs according to international guidelines regardless of the study's allocation. Standard CVD care includes referral to cardiac rehabilitation, which includes a structured exercise regime, and education about CVD risk factors such as stress, dietary modifications and tobacco smoking cessation. All study participants are permitted to seek psychopharmacology, which will be monitored, from outside the study.

\section{Procedure}

The potentially eligible patients are being identified at the hospital 1 to 4 days after their CVD admission by an authorized hospital staff member employed as a trial coordinator in the cardiology department. A pool of eligible participants will be determined by the trial coordinator's review of cardiology admissions and medical records if required. In the first instance, persons will be approached on the hospital ward by the trial coordinator and provided with the study information sheet and an opportunity to discuss any aspects of the study with the trial coordinator. The potentially eligible patients are recontacted by the study's trial coordinator by letter and then telephone 2 to 8 weeks after their CVD admission.
Eligible and consenting participants will provide written informed consent at the baseline appointment. Determination of the depression and anxiety thresholds will be made at baseline. Randomization and patient allocation to trial arms will take place by means of a random number generator prepared in advance by an independent statistician offsite. All participants undergo active monitoring for CVD events and mental well-being (PHQ-9 and GAD-7) through a scheduled phone call every 4 to 6 weeks after randomization. The numbers of eligible and successfully recruited patients will be monitored and discussed on a weekly basis between the $\mathrm{CI}$ and trial manager.

\section{Patient rated measures}

Psychosocial outcomes will be assessed with a battery of fully validated measures at baseline, after the intervention completion (12 to 18 weeks) and during the 6-month follow-up period. The timing of assessments is shown in Table 2 . The self-rated measures will be collected to provide the standard deviation of patient outcomes, which is necessary to perform a power calculation in a larger more definitive trial. Patient rates of alcohol and tobacco use, physical activity, and hospital readmissions will be collected to provide an estimate for a larger more definitive trial.

\section{Anxiety, depression and stress symptoms}

Generalized anxiety symptoms will be measured by the 7item Generalized Anxiety Disorder scale (GAD-7). The

Table 2 Assessment schedule for participants through the study

\begin{tabular}{|c|c|c|c|c|c|c|c|c|}
\hline & & \multirow[b]{2}{*}{ Eligibility } & \multicolumn{6}{|c|}{ Timing of assessment for all participants } \\
\hline & & & Pre-random ization & 4 weeks & 8 weeks & 12 weeks & 18 weeks & 6 months \\
\hline Variable & Measure & & & & & & & \\
\hline \multicolumn{9}{|l|}{ Inclusion criteria } \\
\hline Depression & PHQ-9 & $\checkmark$ & & $\checkmark$ & $\checkmark$ & $\checkmark$ & $\checkmark$ & $\checkmark$ \\
\hline Generalized anxiety & GAD-7 & $\checkmark$ & & $\checkmark$ & $\checkmark$ & $\checkmark$ & $\checkmark$ & $\checkmark$ \\
\hline Psychiatric diagnosis & MINI & $\checkmark$ & & & & & $\checkmark$ & $\checkmark$ \\
\hline \multicolumn{9}{|l|}{ Post-eligibility } \\
\hline Anxiety severity & OASIS & & $\checkmark$ & & & & $\checkmark$ & $\checkmark$ \\
\hline General stress & DASS-21 & & $\checkmark$ & & & & $\checkmark$ & $\checkmark$ \\
\hline $\mathrm{QOL}$ & SF-12 & & $\checkmark$ & & & & $\checkmark$ & $\checkmark$ \\
\hline CVD outcome & MACE & & & & & & $\checkmark$ & $\checkmark$ \\
\hline Physical activity & Exercise & & $\checkmark$ & & & & $\checkmark$ & $\checkmark$ \\
\hline GATS & Tobacco & & $\checkmark$ & & & & $\checkmark$ & $\checkmark$ \\
\hline AUDIT-C & Alcohol & & $\checkmark$ & & & & $\checkmark$ & $\checkmark$ \\
\hline MOS SAS & Adherence & & $\checkmark$ & & & & & \\
\hline $\begin{array}{l}\text { Psychiatric service, medication usage, } \\
\text { satisfaction with care }\end{array}$ & Self-report, audit & & $\checkmark$ & & & & $\checkmark$ & $\checkmark$ \\
\hline
\end{tabular}

AUDIT-C, Alcohol Use Disorders Identification Test-Shortened Clinical Version; CVD, cardiovascular disease; DASS-21, Depression, Anxiety Stress Scales; GAD-7, Generalized Anxiety Disorder-7; GATS, Global Adult Tobacco Survey; MACE, major adverse cardiac event; MINI, MINI International Neuropsychiatric Interview; MOS SAS, Medical Outcomes Study Specific Adherence Scale; OASIS, Overall Anxiety Severity And Impairment Scale; PHQ-9, Patient Health Questionnaire-9; SF-12, Medical Outcomes Study Short Form-12; QOL, quality of life; 
GAD-7 severity threshold for clinically relevant symptoms is a total GAD score $\geq 7$ [52]. This questionnaire has favorable validity as a tool to identify depression and anxiety disorders in medical patients [52]. The Overall Anxiety and Severity Impairment Scale (OASIS) is a five-item brief measure of anxiety symptoms, avoidance, and severity used in a number of large RCTs [56]. Scores $>8$ are indicative of severe anxiety. Depression symptoms will be measured by the 9-item Patient Health Questionnaire (PHQ-9). The PHQ-9 severity threshold for clinically relevant symptoms is a total PHQ score $\geq 10$ [52]. Stress will be measured by the Depression, Anxiety and Stress Scales, a 21-item clinical measure commonly used in Australia, validated in adults to age 90 years $[57,58]$, and in previous studies in the cardiac surgery population $[59,60]$. Mild distress for the Stress scale is $>8$.

\section{Other rating scales}

Quality of life will be assessed with the SF-12. The SF-12 is a commonly utilized and generalizable measure of QOL in CVD populations [61, 62]. Other behavioral factors such as smoking and alcohol use are pertinent to cardiovascular functioning and are likely to be EDBs targeted by the UP intervention. Lifetime and current tobacco use will be measured by items from the Global Adult Tobacco Survey [63]. Recent alcohol use will be measured by the Alcohol Use Disorders Identification Test-Shortened Clinical Version, which provides favorable sensitivity and specificity for the detection of problematic drinking [64]. The physical activity questions from the Australian National Health Surveys are used to classify participants as sedentary or having low, moderate, or high levels of physical activity calculated with metabolic equivalents [65].

\section{Psychiatric disorders}

The MINI is a brief structured interview that will be performed by allocation-naïve assessors to determine the primary psychiatric diagnosis and remission at the end of the study [66, 67]. Assessment takes 20 to 40 minutes to complete and has been validated among cardiac patients [68].

\section{Medical and demographic data}

Demographic, comorbidity and CVD condition data will be obtained via project-specific questions. A statewide hospital registry will be used to identify CVD and psychiatric hospital admissions at 6-month follow-up. Major CVD events include myocardial infarction, stroke, coronary revascularization, cardiac failure, and arrhythmia determined with relevant International Classification of Disease Criteria Codes I00-I99 [69]. Admissions for primary psychiatric causes include suicide attempt, deliberate self-harm, and panic disorder (International Classification of Disease Criteria Codes F00-F99) [69]. Electronic data linkage will be used to determine admissions, and adjudication of study outcomes will be performed by an independent panel of cardiologists, blinded to the randomization arm. Patient confidentiality will be maintained.

\section{Intervention acceptability and feasibility}

Participants will be asked to rate how effective different mental health care treatments were. The questions will assess psychologist treatments (a component of CHAMPS) and outside treatments: specifically, psychiatrist, GP counselling, anti-depressant, anti-anxiety medications, and other areas of support.

\section{Stopping rules}

If there are concerns about significant emerging psychiatric symptoms (for example, psychosis, mania or other serious mental health conditions) associated with risks to the participant or others, and particularly if the PHQ score is $\geq 20$ or the PHQ-9 item 9 score $\geq 2$, the Mental Health Triage will necessarily be contacted, or alternatively, referral to an emergency department will be undertaken if a risk is imminent and high. If significant symptoms or comorbidities (for example, psychosis, hypomania, or substance use) that do not meet the previous criteria emerge at any point during the study, the psychologist will stop the study protocol, treat these conditions and/or provide appropriate referral for psychiatric or drug and alcohol service review. Psychiatric referral should be considered if there are significant risk factors for self-harm or CBT resistance such as personality disorder, past self-harm attempts or treatment-resistant depression.

\section{Ethical considerations}

The study has been approved by Human Research Ethics Committee of The Queen Elizabeth Hospital (approval \#HREC/15/TQEH47). Written informed consent will be obtained from the participants before inclusion. Study participants are free to withdraw from the study at any time. The study carries a suicide risk, and therefore, the patients' levels of suicidal ideation will be monitored with the PHQ-9 (every 4 weeks). As in our real-world clinic, we will follow a protocol for managing suicidal intent and self-harm attempts [38]. An independent data monitoring board will monitor adverse events (cardiac, psychiatric, and other) and report these to the governing ethics board. No plans are made to audit the conduct of the trial until it is completed.

\section{Discussion}

The proposed feasibility RCT intervention for emotional disorders will provide an innovative treatment that potentially better aligns with the complex mental health 
needs for persons afflicted with CVDs. This is perhaps the first investigation of its kind to comprehensively treat a broad range of emotional disorders in CVDs utilizing a single set of therapeutic principles. If the UP is feasible and acceptable, we will develop a larger more definitive trial, and therefore, the findings may hold relevance for the design of future trials, healthcare service delivery, and funding in cardiology.

Several limitations of this trial exist, including that it is applied to multiple emotional disorders, and potential heterogeneity will exist in the study participants' treatment needs. In addition, the findings will require replication, perhaps in larger numbers or more homogenous samples.

At the feasibility stage of intervention development, the establishment of superiority of the UP from TAU is important. Definitive identification of the specific components of the active treatment that fosters change in anxiety, depression, and quality of life among CVD patients will not be possible. Moreover, the length of follow-up should be extended in a larger trial to determine the effects on CVD events.

The present feasibility RCT is designed to evaluate the UP targeting emotional disorder processes in a CVD population, with particular focus on acceptability, acceptance rates, satisfaction with care and attrition. If the trial is viable, it opens up the possibility for interventions to target broader emotional processes in the precarious population with CVD and emotional distress and paves the way for a larger more definitive trial. With increasing recognition of the role of negative emotions in CVDs, the need for depression disorder-only treatments could become increasingly obsolete in CVDs as developments in treatments match those in clinical psychology [46].

\section{Trial status}

The trial has been approved and is currently recruiting.

\begin{abstract}
Abbreviations
CVD: cardiovascular disease; CHAMPS: Cardiovascular Health in Anxiety or Mood Problems Study; EDB: emotion-driven behavior; EUC: enhanced usual care; GAD: generalized anxiety disorder; MACE: major adverse cardiac event; MINI: MINI International Neuropsychiatric Interview; OASIS: Overall Anxiety Severity And Impairment Scale; PHQ: Patient Health Questionnaire; QOL: quality of life; UP: unified protocol.
\end{abstract}

\section{Competing interests}

The authors declare that they have no competing interests.

\section{Authors' contributions}

PJT, DAT, JDH, JFB, TS, EM, BTB, SS-Z, HB, SC, and GAW contributed to the conception and design of the study. PJT, DAT, JDH, JFB, TS, EM, BTB, SS-Z, $\mathrm{HB}, \mathrm{SC}$, and GAW critically revised the manuscript for important intellectual content. PJT, DAT, JDH, JFB, TS, EM, BTB, SS-Z, HB, SC, and GAW made a significant contribution to the research and the development of the manuscript and approved the final version for publication.

\section{Acknowledgement}

The authors wish to thank Marilyn Black and Marg McGee for their assistance with CHAMPS. This study is supported by a Vanguard grant from the National Heart Foundation of Australia and a Menzies Foundation Allied
Health Scholars start-up grant. PJT is supported by the National Health and Medical Research Council of Australia (Neil Hamilton Fairley-Clinical Overseas Fellowship \#1053578). The article processing charge was funded by the Open Access publication fund of the Albert Ludwigs University of Freiburg. The funders had no role in study design, data collection and analysis, decision to publish, or preparation of the manuscript. The authors have no other conflict of interest or competing interests to declare.

\section{Author details}

${ }^{1}$ Department of Rehabilitation Psychology and Psychotherapy, Institute of Psychology, University of Freiburg, Engelbergerstr. 41, Freiburg 79085, Germany. ${ }^{2}$ INSERM, U897-Epidemiology and Biostatistics, Bordeaux, France. ${ }^{3}$ School of Psychology, The University of Adelaide, Adelaide, Australia. ${ }^{4}$ Department of Cardiology, Basil Hetzel Institute, The Queen Elizabeth Hospital and The University of Adelaide, Adelaide, Australia. ${ }^{5}$ Discipline of Psychiatry, The University of Adelaide, Adelaide, Australia. ${ }^{6}$ Department of Psychiatry, The Queen Elizabeth Hospital, Woodville West, Australia. ${ }^{7}$ Center for Anxiety \& Related Disorders, Department of Psychology, Boston University, Boston, USA. ${ }^{8}$ Freemasons Foundation Centre for Men's Health, Discipline of Medicine, School of Medicine, The University of Adelaide, Adelaide, Australia.

Received: 27 May 2015 Accepted: 8 December 2015

Published online: 11 January 2016

\section{References}

1. Barth J, Schumacher M, Herrmann-Lingen C. Depression as a risk factor for mortality in patients with coronary heart disease: a meta-analysis. Psychosom Med. 2004;66:802-13.

2. Samartzis L, Dimopoulos S, Tziongourou M, Nanas S. Effect of psychosocial interventions on quality of life in patients with chronic heart failure: A meta-analysis of randomized controlled trials. J Cardiac Failure. 2013;19:125-34.

3. Baumeister $H$, Haschke A, Munzinger M, Hutter N, Tully PJ. Inpatient and outpatient costs in patients with coronary artery disease and mental disorders: A systematic review. Biopsychosocial Med. 2015;9:11.

4. Baumeister H, Hutter N, Bengel J. Psychological and pharmacological interventions for depression in patients with coronary artery disease. Coch Data Syst Rev. 2011;9:CD008012.

5. Frasure-Smith N, Lespérance F. Depression and anxiety as predictors of 2-year cardiac events in patients with stable coronary artery disease. Arch Gen Psychiatry. 2008;65:62-71.

6. Martens EJ, de Jonge P, Na B, Cohen BE, Lett H, Whooley MA. Scared to death? Generalized anxiety disorder and cardiovascular events in patients with stable coronary heart disease: The Heart and Soul Study. Arch Gen Psychiatry. 2010;67:750-8.

7. Edmondson D, Richardson S, Falzon L, Davidson KW, Mills MA, Neria Y. Posttraumatic stress disorder prevalence and risk of recurrence in acute coronary syndrome patients: a meta-analytic review. PLoS ONE. 2012;7:e38915.

8. Dao TK, Chu D, Springer J, Gopaldas RR, Menefee DS, Anderson T, et al. Clinical depression, posttraumatic stress disorder, and comorbid depression and posttraumatic stress disorder as risk factors for in-hospital mortality after coronary artery bypass grafting surgery. J Thorac Cardiovasc Surg. 2010;140:606-10.

9. Chen YH, Tsai SY, Lee HC, Lin HC. Increased risk of acute myocardial infarction for patients with panic disorder: a nationwide population-based study. Psychosom Med. 2009;71:798-804.

10. Chen YH, Lin HC. Patterns of psychiatric and physical comorbidities associated with panic disorder in a nationwide population-based study in Taiwan. Acta Psychiatr Scand. 2011;123:55-61.

11. Tully PJ, Baune BT. Comorbid anxiety disorders alter the association between cardiovascular diseases and depression: the German National Health Interview and Examination Survey. Soc Psych Psychiatr Epidemiol. 2014:49:683-91.

12. Allonier C, Chevalier A, Zins M, Catelinois O, Consoli SM, Goldberg M, et al. Anxiety or depressive disorders and risk of ischaemic heart disease among French power company employees. Int J Epidemiol. 2004;33:779-86.

13. Scherrer JF, Chrusciel T, Zeringue A, Garfield LD, Hauptman PJ, Lustman PJ, et al. Anxiety disorders increase risk for incident myocardial infarction in depressed and nondepressed Veterans Administration patients. Am Heart J. 2010;159:772-9. 
14. Phillips AC, Batty GD, Gale CR, Deary IJ, Osborn D, Maclntyre K, et al. Generalized anxiety disorder, major depressive disorder, and their comorbidity as predictors of all-cause and cardiovascular mortality: the Vietnam experience study. Psychosom Med. 2009;71:395-403.

15. Tully PJ, Cosh SM, Baumeister $H$. The anxious heart in whose mind? A systematic review and meta-regression of factors associated with anxiety disorder diagnosis, treatment and morbidity risk in coronary heart disease. J Psychosom Res. 2014;77:439-48.

16. Watson D. Differentiating the mood and anxiety disorders: a quadripartite model. Ann Rev Clin Psychol. 2009;5:221-47.

17. Kessler RC, Chiu WT, Demler O, Merikangas KR, Walters EE. Prevalence, severity, and comorbidity of 12-month DSM-IV disorders in the National Comorbidity Survey Replication. Arch Gen Psychiatry. 2005;62:617-27.

18. Dreher H. Psychosocial factors in heart disease: a process model. Adv Mind Body Med. 2004;20:20-31.

19. Suls J, Bunde J. Anger, anxiety, and depression as risk factors for cardiovascular disease: the problems and implications of overlapping affective dispositions. Psychol Bull. 2005;131:260-300.

20. Pogosova N, Saner H, Pedersen SS, Cupples ME, McGee H, Höfer S, et al. Psychosocial aspects in cardiac rehabilitation: From theory to practice. A position paper from the Cardiac Rehabilitation Section of the European Association of Cardiovascular Prevention and Rehabilitation of the European Society of Cardiology. Eur J Prev Cardiol. 2015;22:1290-306.

21. Ladwig KH, Lederbogen F, Albus C, Angermann C, Borggrefe M, Fischer D, et al. Position paper on the importance of psychosocial factors in cardiology: Update 2013. German Med Sci. 2014;12:Doc09.

22. Tully PJ, Winefield H, Baker RA, Winefield H, Denollet J, Pedersen SS, et al. Depression, anxiety and major adverse cardiovascular and cerebrovascular events in patients following coronary artery bypass graft surgery: a five year longitudinal cohort study. Biopsychosocial Med. 2015;9:14.

23. Tully PJ, Baumeister H. Collaborative care for comorbid depression and coronary heart disease: a systematic review and meta-analysis of randomized controlled trials. Syst Rev. 2014;3:127. doi:10.1186/2046-4053-3-127.

24. Rutledge T, Reis VA, Linke SE, Greenberg BH, Mills PJ. Depression in heart failure a meta-analytic review of prevalence, intervention effects, and associations with clinical outcomes. J Am Coll Cardiol. 2006:48:1527-37.

25. Baumeister $\mathrm{H}$, Hutter N, Bengel J. Psychological and pharmacological interventions for depression in patients with diabetes mellitus-a systematic Cochrane review. Diabet Med. 2014;31:773-86.

26. Dickens C, Cherrington A, Adeyemi I, Roughley K, Bower P, Garrett C, et al. Characteristics of psychological interventions that improve depression in people with coronary heart disease: a systematic review and metaregression. Psychosom Med. 2013;75:211-21.

27. Rollman BL, Belnap BH, LeMenager MS, Mazumdar S, Houck PR, Counihan PJ, et al. Telephone-delivered collaborative care for treating post-CABG depression: a randomized controlled trial. J Am Med Assoc. 2009;302:2095-103.

28. Davidson KW, Rieckmann N, Clemow L, Schwartz JE, Shimbo D, Medina V, et al. Enhanced depression care for patients with acute coronary syndrome and persistent depressive symptoms: coronary psychosocial evaluation studies randomized controlled trial. Arch Internal Med. 2010;170:600-8.

29. Ye S, Shaffer JA, Rieckmann N, Schwartz JE, Kronish IM, Ladapo JA, et al. Long-term outcomes of enhanced depression treatment in patients with acute coronary syndromes. Am J Med. 2014;127:1012-6.

30. Fleet $\mathrm{R}$, Lavoie $\mathrm{K}$, Beitman BD. Is panic disorder associated with coronary artery disease? A critical review of the literature. J Psychosom Res. 2000;48:347-56.

31. Katerndahl DA. The association between panic disorder and coronary artery disease among primary care patients presenting with chest pain: an updated literature review. J Clin Psychiatry. 2008;10:276-85.

32. Sardinha A, Nardi AE, Zin WA. Are panic attacks really harmless? The cardiovascular impact of panic disorder. Rev Bras Psiquiatr. 2009;31:57-62.

33. Walters K, Rait G, Petersen I, Williams R, Nazareth I. Panic disorder and risk of new onset coronary heart disease, acute myocardial infarction, and cardiac mortality: cohort study using the general practice research database. Eur Heart J. 2008;29:2981-8.

34. Tully PJ, Turnbull DA, Beltrame JF, Horowitz JD, Cosh S, Baumeister H, et al. Panic disorder and incident coronary heart disease: a systematic review and meta-regression in 1,131,612 persons and 58,111 cardiac events. Psychol Med. 2015:45:2909-20.

35. Wulsin L, Liu T, Storrow A, Evans S, Dewan N, Hamilton C. A randomized, controlled trial of panic disorder treatment initiation in an emergency department chest pain center. Ann Emerg Med. 2002;39:139-43.
36. Lichtman JH, Bigger Jr JT, Blumenthal JA, Frasure-Smith N, Kaufmann PG, Lesperance $F$, et al. Depression and coronary heart disease: recommendations for screening, referral, and treatment: a science advisory from the American Heart Association Prevention Committee of the Council on Cardiovascular Nursing, Council on Clinical Cardiology, Council on Epidemiology and Prevention, and Interdisciplinary Council on Quality of Care and Outcomes Research: endorsed by the American Psychiatric Association. Circulation. 2008;118:1768-75.

37. Huffman JC, Beach SR, Suarez L, Mastromauro CA, Dubois CM, Celano CM, et al. Design and baseline data from the Management of Sadness and Anxiety in Cardiology (MOSAIC) randomized controlled trial. Contemp Clin Trials. 2013;36:488-501.

38. Tully PJ, Wittert GA, Selkow T, Baumeister $H$. The real world mental health needs of heart failure patients are not reflected by the depression Randomized Controlled Trial Evidence. PLoS ONE. 2014;9:e85928.

39. Celano CM, Mastromauro CA, Lenihan EC, Januzzi JL, Rollman BL, Huffman JC. Association of baseline anxiety with depression persistence at 6 months in patients with acute cardiac illness. Psychosom Med. 2012;74:93-9.

40. Hasnain M, Vieweg WR, Lesnefsky EJ, Pandurangi AK. Depression screening in patients with coronary heart disease: a critical evaluation of the AHA guidelines. J Psychosom Res. 2011;71:6-12.

41. Norlund F, Olsson EM, Burell G, Wallin E, Held C. Treatment of depression and anxiety with internet-based cognitive behavior therapy in patients with a recent myocardial infarction (U-CARE Heart): study protocol for a randomized controlled trial. Trials. 2015;16:154

42. Bullis JR, Fortune MR, Farchione TJ, Barlow DH. A preliminary investigation of the long-term outcome of the Unified Protocol for Transdiagnostic Treatment of Emotional Disorders. Compr Psychiatry. 2014;55:1920-7.

43. Gallagher MW, Sauer-Zavala SE, Boswell JF, Carl JR, Bullis J, Farchione TJ, et al. The impact of the unified protocol for emotional disorders on quality of life. Int J Cogn Ther. 2013;6:57-72.

44. Farchione TJ, Fairholme CP, Ellard KK, Boisseau CL, Thompson-Hollands J, Carl JR, et al. Unified Protocol for Transdiagnostic Treatment of Emotional Disorders: A Randomized Controlled Trial. Behav Ther. 2012;43:666-78.

45. Barlow DH, Farchione TJ, Fairholme CP, Ellard KK, Boisseau CL, Allen LB, et al. The unified protocol for transdiagnostic treatment of emotional disorders: Therapist quide. New York: NY Oxford University Press; 2011.

46. Ellard KK, Fairholme CP, Boisseau CL, Farchione TJ, Barlow DH. Unified Protocol for the Transdiagnostic Treatment of Emotional Disorders: Protocol Development and Initial Outcome Data. Cogn Behav Pract. 2010;17:88-101.

47. Sauer-Zavala S, Boswell JF, Gallagher MW, Bentley KH, Ametaj A, Barlow DH. The role of negative affectivity and negative reactivity to emotions in predicting outcomes in the unified protocol for the transdiagnostic treatment of emotional disorders. Behav Res Ther. 2012;50:551-7.

48. Tully PJ. A good time to panic? Premorbid and postmorbid panic disorder in heart failure affects cardiac and psychiatric cause admissions. Australasian Psychiatry. 2015;23:124-7.

49. Tully PJ, Selkow T, Bengel J, Rafanelli C. A dynamic view of comorbid depression and generalized anxiety disorder symptom change in chronic heart failure: discrete effects of cognitive behavioral therapy, exercise rehabilitation, and psychotropic medication. Disabil Rehab. 2015;37:585-92.

50. Zwarenstein M, Treweek S, Gagnier JJ, Altman DG, Tunis S, Haynes B, et al. Improving the reporting of pragmatic trials: an extension of the CONSORT statement. BMJ. 2008;337:a2390.

51. Colquhoun DM, Bunker SJ, Clarke DM, Glozier N, Hare DL, Hickie IB, et al. Screening, referral and treatment for depression in patients with coronary heart disease. Med J Aust. 2013;198:483-4.

52. Kroenke K, Spitzer RL, Williams JB, Lowe B. The Patient Health Questionnaire Somatic, Anxiety, and Depressive Symptom Scales: a systematic review. Gen Hosp Psychiatry. 2010;32:345-59.

53. Barlow DH, Farchione TJ, Fairholme CP, Ellard KK, Boisseau CL, Allen LB, et al. The Unified Protocol for Transdiagnostic Treatment of Emotional Disorders: Client Workbook. New York: Oxford University Press; 2011.

54. beyondblue: Coronary heart disease, anxiety and depression. Edited by beyondblue, vol. Fact Sheet 18.

55. Tully PJ, Higgins R. Depression screening, assessment and treatment for patients with coronary heart disease: a review for psychologists. Aust Psychol. 2014:49:337-44.

56. Campbell-Sills L, Norman SB, Craske MG, Sullivan G, Lang AJ, Chavira DA, et al. Validation of a brief measure of anxiety-related severity and impairment: the Overall Anxiety Severity and Impairment Scale (OASIS). J Affect Disord. 2009;112:92-101. 
57. Crawford J, Cayley C, Lovibond PF, Wilson PH, Hartley C. Percentile Norms and Accompanying Interval Estimates from an Australian General Adult Population Sample for Self-Report Mood Scales (BAI, BDI, CRSD, CES-D, DASS, DASS-21, STAI-X, STAI-Y, SRDS, and SRAS). Aust Psychol. 2011;46:3-14.

58. Lovibond PF, Lovibond SH. The structure of negative emotional states: comparison of the Depression Anxiety Stress Scales (DASS) with the Beck Depression and Anxiety Inventories. Behav Res Ther. 1995;33:335-43.

59. Tully PJ, Baker RA, Knight JL, Turnbull DA, Winefield HR. Neuropsychological function 5 years after cardiac surgery and the effect of psychological distress. Arch Clin Neuropsychol. 2009;24:741-51.

60. Tully PJ, Baker RA, Turnbull DA, Winefield HR, Knight JL. Negative emotions and quality of life six months after cardiac surgery: the dominant role of depression not anxiety symptoms. J Behav Med. 2009;32:510-22.

61. Tully PJ. Quality-of-Life measures for cardiac surgery practice and research: a review and primer. J Extra Corpor Technol. 2013:45:8-15.

62. Muller-Nordhorn J, Roll S, Willich SN. Comparison of the short form (SF)-12 health status instrument with the SF-36 in patients with coronary heart disease. Heart. 2004;90:523-7.

63. Global Adult Tobacco Survey Collaborative Group. Tobacco questions for surveys: a subset of key questions from the Global Adult Tobacco Survey, vol. 2. Atlanta, GA: Centers for Disease Control and Prevention; 2011.

64. Bush K, Kivlahan DR, McDonell MB, Fihn SD, Bradley KA. The AUDIT alcoho consumption questions (AUDIT-C): an effective brief screening test for problem drinking. Ambulatory Care Quality Improvement Project (ACQUIP). Alcohol Use Disorders Identification Test. Arch Intern Med. 1998;158:1789-95.

65. Australian Bureau of Statistics. National Health Survey: users' guide. Canberra, Australia: Australian Bureau of Statistics; 2003.

66. Amorim P, Lecrubier Y, Weiller E, Hergueta T, Sheehan D. DSM-III-R Psychotic Disorders: procedural validity of the Mini International Neuropsychiatric Interview (MINI). Concordance and causes for discordance with the CIDI. Eur Psychiatry. 1998;13:26-34.

67. Sheehan DV, Lecrubier Y, Sheehan K, Amorim P, Janavs J, Weiller E, et al. The Mini-International Neuropsychiatric Interview (M.I. N.I.): the development and validation of a structured diagnostic psychiatric interview for DSM-IV and ICD-10. J Clin Psychiatry. 1998:59(Supplement 20):22-33.

68. Tully PJ, Penninx BW. Depression and anxiety among coronary heart disease patients: can affect dimensions and theory inform diagnostic disorder based screening? J Clin Psychol. 2012;68:448-61.

69. World Health Organization: International Statistical Classification of Diseases and Related Health Problems 10th Revision Version for 2007 Available online at http://www.who.int/classifications/apps/icd/icd10online/. 2007. Accessed 15 Apr 2015.

\section{Submit your next manuscript to BioMed Central and we will help you at every step:}

- We accept pre-submission inquiries

- Our selector tool helps you to find the most relevant journal

- We provide round the clock customer support

- Convenient online submission

- Thorough peer review

- Inclusion in PubMed and all major indexing services

- Maximum visibility for your research

Submit your manuscript at www.biomedcentral.com/submit

C Biomed Central 